ИЗВЕСТИЯ АКАДЕМИИ НАУК ЭСТОНСКОЙ ССР. ТОМ 21 ХИМИЯ * ГЕОЛОГИЯ. 1972 , № 3

\title{
ИСПОЛЬЗОВАНИЕ ТЕОРИИ РЕГУЛЯРНЫХ РАСТВОРОВ ДЛЯ ОПИСАНИЯ РАСПРЕДЕЛЕНИЯ ФЕНОЛОВ И АРОМАТИЧЕСКИХ АМИНОВ МЕЖДУ ФАЗАМИ ВОДЫ И ОРГАНИЧЕСКОГО РАСТВОРИТЕЛЯ
}

В литературе опубликовано много данных о распределении органических соединений между фазами воды и органических растворителей разного строения. В некоторых работах по равновесию жидкость-жидкость сделана попытка установить эмпирическую зависимость между экстрагирующей способ̆остью экстрагента и его диэлектрической постоянной $\left[{ }^{1-7}\right]$, параметром растворимости Гильдебранда $\left[{ }^{5-7}\right]$, молярным объемом [7], а также параметром Кирквуда-Бауера-Мага [2-4]. Указано также на существование корреляции между коэффициентом распределения и взаимной растворимостью фаз [ $\left.{ }^{6}\right]$ или межфазовым поверхностным натяжением [8]. В работах [9-12] предложен эмпирический, но простой и надежный метод для предсказания коэффициента распределения определенного класса веществ на основании данных о распределенин «стандартного» (для данного класса) соединения.

В настоящей работе сделана попытка использовать для описания межфазового распределения ароматических гидроксил- и азотсодержащих соединений теорию регулярных растворов Дж. Х. Гильдебранда и Р. Л. Скотта $\left[{ }^{13}\right]$. При этом водная фаза условно рассматривается как регулярная, а взаимодействие между распределяемым веществом и водой учитывается путем введения эмпирического параметра растворимости для водной фазы. Такой подход ранее был предложен Т. Вакахайаши и др. $\left[{ }^{14-17}\right]$ для корреляции распределения некоторых хелатизированных соединений и недавно использован И. Койма и др. [18] также для карбоновых кислот.

\section{Модель для описания равновесия при распределении фенолов}

Распределение фенолов $(\mathrm{ArOH})$ между водой и органическим растворителем, не образующим с распределяемым веществом водородных связей, определяется следующими равновесными реакциями; 
1. «Физическое» распределение мономера

$$
\mathrm{ArOH}_{(\mathrm{o})} \rightleftarrows \mathrm{ArOH}_{(\mathrm{B})}
$$

с константой равновесия

$$
\mathrm{K}_{\mathrm{p}}=\frac{[\mathrm{ArOH}]_{(\mathrm{o})}}{[\mathrm{ArOH}]_{(\mathrm{B})}},
$$

где индексы (о) и (в) относятся соответственно к органической и водной фазам.

2. Самоассоциация в органической фазе

$$
\begin{gathered}
2 \mathrm{ArOH}_{(0)} \rightleftarrows(\mathrm{ArOH})_{2(0)}, \quad 3 \mathrm{ArOH}_{(0)} \rightleftarrows(\mathrm{ArOH})_{3(0)}, \ldots, \\
\mathrm{L} \cdot \mathrm{ArOH}_{(0)} \rightleftarrows(\mathrm{ArOH})_{\mathrm{L}(0)}
\end{gathered}
$$

с константами равновесия соответственно

$$
K_{12}=\frac{\left[(\mathrm{ArOH})_{2}\right]_{(0)}}{[\mathrm{ArOH}]_{(0)}^{2}}, K_{13}=\frac{\left[(\mathrm{ArOH})_{3}\right]_{(0)}}{[\mathrm{ArOH}]_{(0)}^{3}}, \ldots, K_{1 \mathrm{~L}}=\frac{\left[(\mathrm{ArOH})_{L}\right]_{(0)}}{[\mathrm{ArOH})]_{(0)}^{\mathrm{L}}} .
$$

3. Диссоциация (ионизация) в водной фазе

$$
\mathrm{ArOH}_{(\mathrm{B})} \rightleftarrows \mathrm{ArO}_{(\mathrm{B})}^{-}+\mathrm{H}_{(\mathrm{B})}^{+}
$$

с константой ионизации

$$
\mathrm{K}_{\text {дисс }}=\frac{\left[\left(\mathrm{ArO}^{-}\right]_{(\mathrm{B})}\left[\mathrm{H}^{+}\right]_{(\mathrm{B})}\right.}{[\mathrm{ArOH}]_{(\mathrm{B})}} .
$$

Кажущийся коэффициент распределения (в мольных долях) выражается

$\mathrm{P}=\frac{\mathrm{x}_{(\mathrm{o})}}{\mathrm{x}_{(\mathrm{B})}}=\frac{\gamma_{(\mathrm{B})}}{\gamma_{(\mathrm{o})}}=\frac{\left.[\mathrm{ArOH}]_{(0)}+2\left[(\mathrm{ArOH})_{2}\right]_{(\mathrm{o})}+3[\mathrm{ArOH})_{3}\right]_{(\mathrm{o})}+\ldots+\mathrm{L}\left[(\mathrm{ArOH})_{\mathrm{L}}\right]_{(0)}}{[\mathrm{ArOH}]_{(\mathrm{B})}+\left[\mathrm{ArO}^{-}\right]_{(\mathrm{B})}}$,

где $\mathrm{x}$ - суммарная (стехиометрическая) концентрация распределяемого вецества (в мольных долях), $\gamma$ - его коэффициент активности.

С учетом (1), (2) и (3) из уравнения (4) путем несложного преоб. разования получаем

$$
\begin{aligned}
\mathrm{P}=\frac{\mathrm{K}_{\mathrm{p}}}{1+\mathrm{K}_{\text {дис c }}\left[\mathrm{H}^{+}\right]_{(\mathrm{B})}^{-1}} & \left(1+2 \mathrm{~K}_{12}[\mathrm{ArOH}]_{(0)}+3 \mathrm{~K}_{13}[\mathrm{ArOH}]_{(0)}^{2}+\ldots+\right. \\
& \left.+\mathrm{LK}_{1 \mathrm{~L}}[\mathrm{ArOH}]_{(0)}^{\mathrm{L}-1}\right) .
\end{aligned}
$$

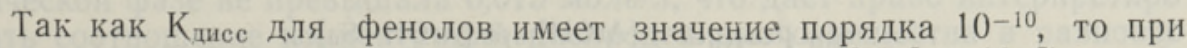
экстракции из кислых и нейтральных растворов $\left(\left[\mathrm{H}^{+}\right] \geqslant 10^{-7}\right)$ диссоциацией фенола можно пренебречь, и

$$
\begin{gathered}
\mathrm{P}=\mathrm{K}_{\mathrm{p}}\left(1+2 \mathrm{~K}_{12} \mathrm{~K}_{\mathrm{p}}[\mathrm{ArOH}]_{(\mathrm{B})}+3 \mathrm{~K}_{13} \mathrm{~K}_{\mathrm{p}}^{2}[\mathrm{ArOH}]_{(\mathrm{B})}^{2}+\ldots+\right. \\
\left.+\mathrm{LK}_{1 \mathrm{~L}} \mathrm{~K}_{\mathrm{p}}^{\mathrm{L}-1}[\mathrm{ArOH}]_{(\mathrm{B})}^{\mathrm{L}-1}\right) .
\end{gathered}
$$

Нетрудно убедиться, что при бесконечно малой концентрации распре деляемого вещества $\left([\mathrm{ArOH}]_{(в)} \rightarrow 0\right)$ P равен «физическому» коэффици- 
енту распределения мономерного вещества $\mathrm{K}_{\mathrm{p}}$ *. Последний определяется параметрами взаимодействия распределяемого вещества с растворителями и численно выражается соотношением его коэффициентов активности в равновесных фазах при бесконечном разбавлении

$$
K_{p}=\left(\gamma_{(B)}\right)_{x \rightarrow 0} /\left(\gamma_{(0)}\right)_{x \rightarrow 0}
$$

С другой стороны, согласно теории регулярных растворов Дж. X. Гильдебранда [13], активность (а) вещества A в растворителе S выражается

$$
\ln \mathrm{a}_{\mathrm{A}}=\ln \varphi_{\mathrm{A}}+\varphi_{\mathrm{s}}\left(1-\frac{\mathrm{V}_{\mathrm{A}}}{\mathrm{V}_{\mathrm{S}}}\right)+\frac{\mathrm{V}_{\mathrm{A}}}{\mathrm{RT}} \varphi_{\mathrm{S}}^{2}\left(\delta_{\mathrm{A}}-\delta_{\mathrm{S}}\right)^{2},
$$

где $\varphi$ - объемная доля, $\mathrm{V}$ - молярный объем, $\delta$ - параметр растворимости Гильдебранда, индексы А и s относятся соответственно к растворенному вешеству и растворитєлю.

Условно написав то же для водной фазы и пренебрегая взаимной растворимостью воды и органического растворителя, получаем для равновесного состояния $\left(\mathrm{a}_{\mathrm{A}(0)}=\mathrm{a}_{\mathrm{A}(\mathrm{B})}\right)$

$$
\begin{gathered}
\ln \frac{\varphi_{A(0)}}{\varphi_{A(B)}}=\left[\varphi_{B}\left(1-\frac{V_{A}}{V_{B}}\right)-\varphi_{o p r}\left(1-\frac{V_{A}}{V_{o p r}}\right)\right]+ \\
+\frac{V_{A}}{R T}\left[\varphi_{B}\left(\delta_{A}-\delta_{B}\right)+\varphi_{o p r}\left(\delta_{A}-\delta_{o p r}\right)\right]\left[\varphi_{B}\left(\delta_{A}-\delta_{B}\right)-\delta_{o p r}\left(\delta_{A}-\delta_{o p r}\right)\right],
\end{gathered}
$$

где индексы А, в и орг относятся соответственно к распределяемому веществу, воде и органическому растворителю.

При бесконечно малой концентрапии распределяемого вещества в обеих фазах

$$
\varphi_{\mathrm{B}}=\varphi_{\text {орг }}=1 \quad \text { и } \quad \varphi_{\mathrm{A}(0)} / \varphi_{\mathrm{A}(\mathrm{B})}=\mathrm{C}_{\mathrm{A}(0)} / \mathrm{C}_{\mathrm{A}(\mathrm{B})}=\mathrm{K}_{\mathrm{p}} \cdot \mathrm{V}_{\mathrm{B}} / \mathrm{V}_{\text {opr }},
$$

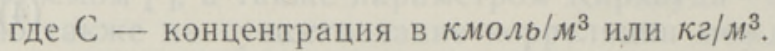

Тогда для объемного коэффициента распределения $\left(\mathrm{k}_{\mathrm{p}}\right)$ получим

$$
\begin{gathered}
\lg k_{p}=\lg \left(K_{p} \cdot V_{B} / V_{o p r}\right)= \\
=\frac{V_{A}}{2,3 R T}\left[\left(\delta_{B}+\delta_{o p r}-2 \delta_{A}\right)\left(\delta_{B}-\delta_{o p r}\right)-R T\left(\frac{1}{V_{B}}-\frac{1}{V_{o p r}}\right)\right] .
\end{gathered}
$$

* Фактически и приведенная схема является улрошенной. Большинство полярных веществ подвергается сольватацни в сдной или обеих фазах. Поэтому

$$
\begin{gathered}
\lim _{[\mathrm{ArOH}]_{(\mathrm{B})} \rightarrow 0} \mathrm{P}=\mathrm{K}_{\mathrm{p}}=\frac{[\mathrm{ArOH}]_{(\mathrm{o})}+\left[\mathrm{ArOH} \cdot \mathrm{S}_{\mathrm{n}}\right]_{(0)}}{[\mathrm{ArOH}]_{(\mathrm{B})}+\left[\mathrm{ArOH} \cdot\left(\mathrm{H}_{2} \mathrm{O}\right)_{\mathrm{m}}\right]_{(\mathrm{B})}}= \\
=\mathrm{K}_{\mathrm{p}}^{\prime}\left(1+\beta_{\mathrm{n}} \mathrm{S}_{(\mathrm{O})}^{\mathrm{n}}\right)\left(1+\beta_{\mathrm{m}}\left[\mathrm{H}_{2} \mathrm{O}\right]_{(\mathrm{B})}^{\mathrm{m}}\right)^{-1} \\
\mathrm{~K}_{\mathrm{p}}=\mathrm{K}_{\mathrm{p}}^{\prime} \alpha_{(0)} \alpha_{(\mathrm{B})}^{-1},
\end{gathered}
$$

где $\mathrm{S}$ - растворитель, $\mathrm{K}_{\mathrm{p}}^{\prime}$ - коэффициент распределения несольватированного мономера, $\beta_{\mathrm{n}}$ и $\beta_{\mathrm{m}}-$ константы образования сольватов $\mathrm{ArOH} \cdot \mathrm{S}_{\mathrm{n}}$ (в органической фазе) и $\mathrm{ArOH} \cdot\left(\mathrm{H}_{2} \mathrm{O}\right)_{\mathrm{m}}$ (в водной фазе), $\alpha_{(0)}$ и $\alpha_{(\text {в) }}-$ суммарные константы, учитывающие химическое взаимодействие (сольватацию) соответственно в органической и водной фазах. 


\section{Обозначив}

$$
\mathrm{Y}=\frac{2,3 \mathrm{RT}}{\mathrm{V}_{\mathrm{A}}} \lg \mathrm{k}_{\mathrm{p}}, \quad \mathrm{A}=\mathrm{RT}\left(\frac{1}{\mathrm{~V}_{\mathrm{B}}}-\frac{1}{\mathrm{~V}_{\mathrm{opr}}}\right) \text { и } \mathrm{Z}=\mathrm{Y}+\mathrm{A},
$$

уравнение (10) можно написать в виде

или

$$
Z=\left(\delta_{\mathrm{B}}+\delta_{\mathrm{opr}}-2 \delta_{\mathrm{A}}\right)\left(\delta_{\mathrm{B}}-\delta_{\mathrm{opr}}\right)
$$

$$
\mathrm{Z} /\left(\delta_{\mathrm{B}}-\delta_{\mathrm{opr}}\right)=\delta_{\mathrm{B}}+\delta_{\mathrm{opr}}-2 \delta_{\mathrm{A}}
$$

H

$$
Z=-\delta_{o p r}^{2}+B_{1} \delta_{o p r}+B_{0}
$$

где $\mathrm{B}_{1}=2 \delta_{\mathrm{A}} \quad$ и $\quad \mathrm{B}_{0}=\delta_{\mathrm{B}}\left(\delta_{\mathrm{B}}-2 \delta_{\mathrm{A}}\right)$.

Коэффициенты уравнения (14) $\mathrm{B}_{0}$-и $\mathrm{B}_{1}$, найденные методом наименьших квадратов из опытных данных по равновесию жидкость-жидкость в системах, где органическая фаза при бесконечном разбавлении подчиняется теории регулярных растворов, дают $\delta_{\mathrm{A}}$ и $\delta_{\mathrm{B}}$, в которых фактическое отклонение поведения водной фазы от теории регулярных растворов учтено чисто эмпирическим путем.

\section{Экспериментальная часть и обработка результатов}

Использованные в работе растворители (исходные препараты марки «ч.» или «х. ч.») перед применением были очищены от примесей способами, предложенными в монографии [19], и ректифицированы. Фенолы очищались путем отгонки из них нейтральных примесей (в щелочной среде) и тщательной ректификации в высокоэффективной колонне. В качестве водной фазы использовался бндистиллят.

При определении коэффициентов распределения равновесие фаз достигалось путем ннтенсивного встряхивания смеси растворителя и водного раствора фенола в термостатированной $\left(20 \pm 1^{\circ} \mathrm{C}\right)$ делительной воронке и последующего расслаивания в теченне 2 ч. После этого равновесную органическую фазу обрабатывали избытком раствора $\mathrm{NaOH}$, полученный фенолят анализировали на фенол (крезол) бромометрически (после отгонки следов растворителя). Из подщелоченной водной фазы отгоняли следы растворителя, после чего раствор анализировали бромометрически. Как правило, расхождения в балансе распределяемого вещества не превышали $\pm 2 \%$. Коэффициенты распределения в параллельных опытах обычно не отличались больше, чем на $2-3 \%$.

Как правило, общая концентрация распределяемого вещества в органической фазе не превышала 0,015 моль/ $\Omega$, что дает право интерпретировать соотношение концентраций распределяемого вещества в равновесных фазах как коэффициент распределения мономера при бесконечном разбавлении ** $\left(\mathrm{K}_{\mathrm{p}}\right)$.

С целью проверки правильности исходных теоретических соображений на более широком экспериментальном материале при обработке

** Для некоторых растворителей исследовалась также зависимость кажущегося коэфффициента распределения от концентрации. Результаты показали, что в области конщентрации до 0,03 моль/л Р мало отлнчается от значения, полученного экстраполя цией до нулевой конщентрации. Это свндетельствует, что в столь разбавленных растворах самоассоциацией распределяемого вещества в первом приближении можно пренебречь. 
результатов, кроме оригинальных данных самих авторов, использовались также данные Я. Коренмана и др. $\left[{ }^{7,20,21}\right]$ и И. Коренмана и др. $\left[{ }^{22,23}\right]$. Коэффициенты распределения для фенола были авторами определены заново, так как данные, опубликованные Я. Коренманом [7, 24, 25], не сходятся с нашими результатами, а также между собой.

Параметры растворимости $\left(\delta_{\text {орг }}\right)$, молярные объемы и параметр А использованных в работе растворителей приводятся в табл. 1. Параметр растворимости при $20^{\circ}$ находили из соотношения $\mathrm{d} \ln \delta_{\text {opr }} / \mathrm{d} \ln \mathrm{V}_{\mathrm{opr}}=$ $=-1,25$ по данным $\left[{ }^{13}\right]$ и $\left[{ }^{26}\right]$, молярный объем - линейной интерполяцией, параметр А -- по (11).

Әкспериментально найденные коэффициенты распределения, а также функция Z (см. уравнение (11)) для исследованных фенолов приведекы в табл. 2. На основании их методом наименьших квадратов рассчитаны коэффициенты $\mathrm{B}_{0}$ и $\mathrm{B}_{1}$ уравнения (14), из которых получены $\delta_{\mathrm{A}}$ и $\delta_{\mathrm{B}}$. В расчетах молярные объемы фенолов $\left(\mathrm{V}_{\mathrm{A}}\right)$ при $20^{\circ}$ приняты (в $c \mu^{3} /$ моль $):$ фенол - 87,7, o-крезол - 103,5, -кррезол - $104,6, n$-крезол $104,5, \alpha$-нафтол $-120,0$.

Таблица 1

Параметры растворимости, молярные объемы и параметр А использованных растворителей при $20^{\circ} \mathrm{C}$

\begin{tabular}{|c|c|c|c|c|}
\hline & Растворитель & $\begin{array}{c}\delta_{\mathrm{opr}} \\
\left(\kappa a \Omega / c \mu^{3}\right)^{1 / 2}\end{array}$ & $\begin{array}{c}\mathrm{V}_{\mathrm{opr}} \\
c \mathrm{M}^{3} / \mathrm{MO} \boldsymbol{}\end{array}$ & $\mathrm{A}$ \\
\hline 1 & Гексан & 7,34 & 130,7 & 27,8 \\
\hline 2 & Гептан & 7,53 & 146,6 & 28,3 \\
\hline 3 & Октан & 7,60 & 162,6 & 28,7 \\
\hline 4 & Нонан & 7,71 & 178,7 & 29,0 \\
\hline 5 & Декан & 7,79 & 194,9 & 29,25 \\
\hline 6 & Циклогексан & 8,18 & 108,1 & 26,85 \\
\hline 7 & Бензол & 9,23 & 88,9 & 25,65 \\
\hline 8 & Толуол & 8,96 & 106,3 & 26,8 \\
\hline 9 & о-Ксилол & 9,05 & 120,6 & 27,4 \\
\hline 10 & м-Ксилол & 8,82 & 122,8 & $27, \overline{5}$ \\
\hline 11 & $n$-Ксилол & 8,71 & 123,3 & $27, \overline{5}$ \\
\hline 12 & Этилбензол & 8,86 & 122,4 & 27,5 \\
\hline 13 & Изопропилбензол & 8,70 & 139,5 & 28,05 \\
\hline 14 & Четыреххлористый & & & \\
\hline & углерод & 8,66 & 96,5 & 26,2 \\
\hline 15 & Хлороформ & $\begin{array}{l}9,28 \\
9,56\end{array}$ & $\begin{array}{r}80,2 \\
1017\end{array}$ & $\begin{array}{l}25,0 \\
26,6\end{array}$ \\
\hline 17 & $\begin{array}{l}\text { Хлорбензол } \\
1,2 \text {-Дихлорэтан }\end{array}$ & 9,85 & 79,0 & 24,85 \\
\hline
\end{tabular}

Результаты расчетов показали, что межфазовое распределение исследованных фенолов, за исключением 0 -крезола, хорошо описывается уравнением (12), если использовать для параметра растворимости водной фазы значение $\delta_{\text {в }}=19,0$, полученное как среднее для фенола, $九$ - и $n$-крезолов и $\alpha$-нафтола. Для них зависимость $Z /\left(\delta_{\text {в }}-\delta_{\text {орг }}\right)$ от $\delta_{\text {opr }}$ (уравне ние (13)) действительнс представляет собой прямую с угловым коэфффи. циентом 1,00 (рис. $1-3$ ). По-видимому, постоянство $\delta_{\text {в }}$ при распределении указанных фенолов свидетельствует о приблизительно одинаковой степени разрушения структуры воды и роли образования Н-связей между фенолом и водой в водной фазе. В случае же 0 -крезола (рис. 4), имеющего приблизительно такой же молярный объем, аналогичная обработка дает $\delta_{\mathrm{B}}=18,05$, что, по мнению авторов, является следствием бо́льшего разрушения структуры воды при гидратацин гидроксильной группы, замещенной в орто-положении. 


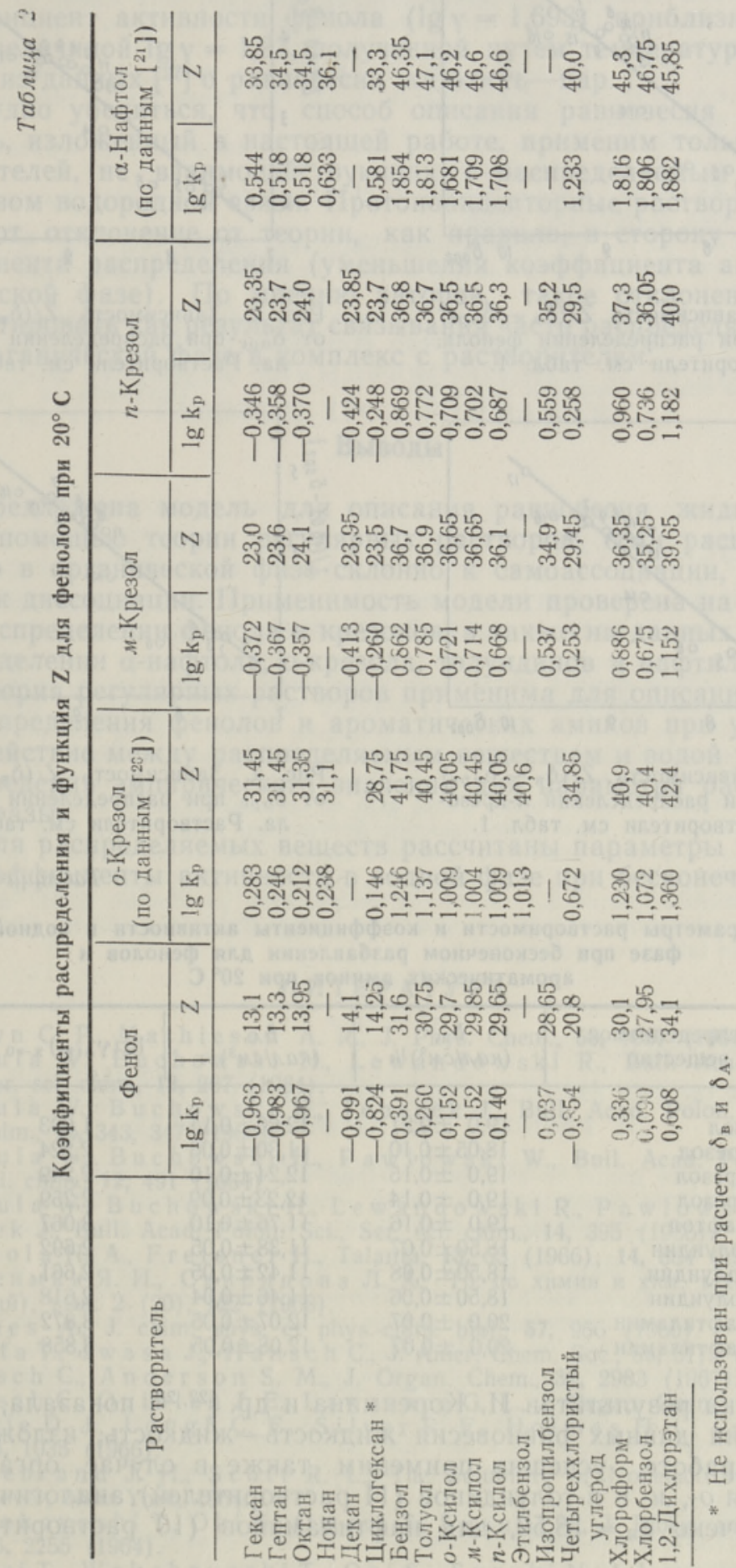




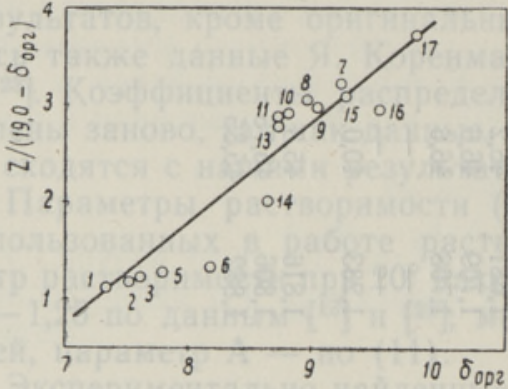

Рис. 1. Зависимость $\mathrm{Z} i\left(\delta_{\mathrm{B}}-\delta_{\text {орг }}\right)$

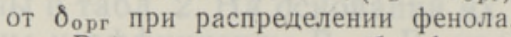

Растворители см. табл. 1.

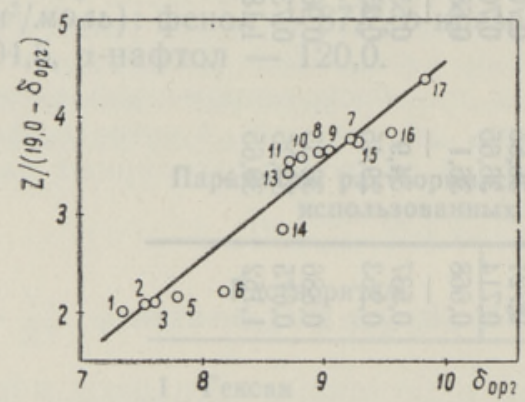

Рис 3. Зависимость $Z /\left(\delta_{\mathrm{B}}-\delta_{\text {орг }}\right)$

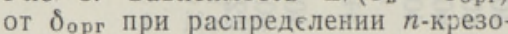
ла. Растворители см. табл. 1.

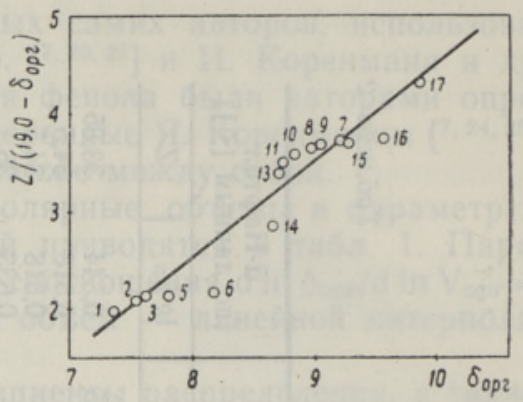

Pис. 2. Зависнмость $Z /\left(\delta_{\mathrm{B}}-\delta_{\text {орг }}\right)$

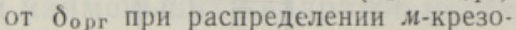
ла. Растворители см. табл. 1.

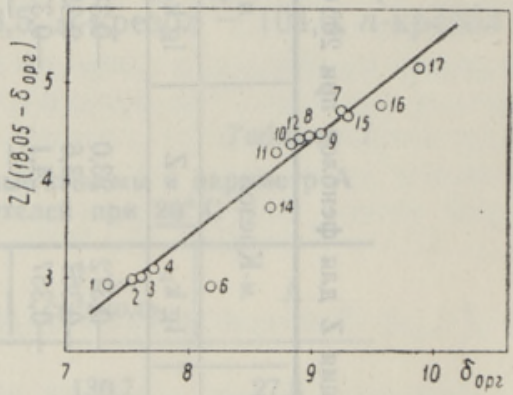

Рис. 4. Зависимость $Z i\left(\delta_{\mathrm{B}}-\delta_{\text {орг }}\right)$

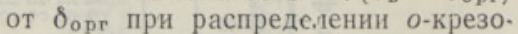
ла. Растворители см. табл. 1.

\section{Таблица 3}

Параметры растворимости и коэффициенты активности в водной фазе при бесконечном разбавлении для фенолов и ароматических аминов при $20^{\circ} \mathrm{C}$

\begin{tabular}{|c|c|c|c|}
\hline $\begin{array}{c}\text { Распределяемое } \\
\text { вещество }\end{array}$ & $\begin{array}{c}\delta_{\mathrm{B}} \\
\left(\kappa a \Omega / \mathrm{CM}^{3}\right)^{1 / 2}\end{array}$ & $\begin{array}{c}\delta_{A}, \\
\left(\kappa a \Omega / C M^{3}\right)^{1 / 2}\end{array}$ & $\lg \left(\gamma_{A(B)}\right)_{x \rightarrow 0}$ \\
\hline Фенол & $19,0 \pm 0,21$ & $12,60 \div 0,14$ & 1,693 \\
\hline o-Крезол & $18,05 \pm 0,10$ & $11,30 \pm 0,06$ & 2,224 \\
\hline M-Крезол & $19,0 \pm 0,15$ & $12,24 \pm 0,10$ & 2,249 \\
\hline$n$-Крезол & $19,0 \pm 0,14$ & $12,23 \pm 0,09$ & 2,259 \\
\hline$\alpha$-Нафтол & $19,0 \pm 0,16$ & $11,76 \pm 0,10$ & 3,067 \\
\hline o-Толуидин & $18,50 \pm 0,07$ & $11,38 \pm 0,05$ & 2,692 \\
\hline м-Толуидин & $18,50 \pm 0,08$ & $11,42 \pm 0,05$ & 2,661 \\
\hline$n$-Толундин & $18,50 \pm 0,06$ & $11,46 \pm 0,04$ & 2,618 \\
\hline$\alpha$-Нафтиламин & $20,0 \pm 0,07$ & $12,07 \pm 0,05$ & 3,872 \\
\hline$\beta$-Нафтиламин & $20,0 \pm 0,07$ & $12,08 \pm 0,05$ & 3,858 \\
\hline
\end{tabular}

Обработка результатов И. Коренмана и др. $\left[{ }^{22,23}\right]$ показала, что способ описания данных равновесия жидкость-жидкость, изложенный в настоящей работе, успешно применим также в случае органических аминов. Для 0 -, $\boldsymbol{M}$ - и $n$-толуидинов (11 растворителей) аналогичным способом получено $\delta_{\mathrm{B}}=18,50$, для нафтиламинов (10 растворителей) $\delta_{\mathrm{B}}=20,0$.

Знание величин $\delta_{A}$ и $\delta_{n}$ дает возможность расчета коэффициента активности распределяемого вещества в водной фазе при бесконечном 
разбавлении по формуле (8) (табл. 3). При этом, конечно, не следует ожидать большой точности. Тем не менее, найденный в настоящей работе коэффициент активности фенола $(\lg \gamma=1,693)$ приблизительно сходится с величиной $\lg \gamma=1,93$, полученной путем температурной экстраполяции из данных $[27]$ о равновесии жидкость-пар.

Нетрудно убедиться, что способ описания равновесия жидкость-. жидкость, изложенный в настоящей работе, прнменим только в случае растворителей, не взаимодействующих с распределяемым веществом посредством водородной связи. Протоноакцепторные растворители обусловливают отклонение от теории, как правило, в сторону увеличения коэффициента распределения (уменьшения коэффициента активности в органической фазе). По мнению авторов, такое отклонение следует интерпретировать как результат связывания части распределяемого вещества в органической фазе в комплекс с растворителем.

\section{Выводы}

1. Предложена модель для описания равновесия жидкость-жидкость с помощью теории регулярных растворов, если распределяемое веілество в органической фазе склонно к самоассоциации, а в водной фазе - к диссоциации. Применимость модели проверена на наших данных о распределении фенола и крезолов, а также на данных литерагуры о распределении $\alpha$-нафтола, 0 -крезола, толуидинов и нафтиламинов.

2. Теория регулярных растворов применима для описания межфазового распределения фенолов и ароматических аминов при условии, что взаимодействие между распределяемым веществом и водой учитывается путем введения эмпирического значения для параметра растворимости водной фазы.

3. Для распределяемых веществ рассчитаны параметры растворимости и коэффициенты активности в водной фазе при бесконечном разбавлении.

\section{Л И Т Е РА Т У РА}

1. Brown C. P., Mathies on A. R., J. Phys. Chem.; 58, 1057 (1954).

2. Kemula W., Buchowski H., Lewandowski R., Bull. Acad. Polon. Sci., Ser. sci. chim., 12, 267 (1964).

3. Kemula W., Buchowski H., Teperek J., Bull. Acad. Polon. Sci., Ser. sci. chim., 12, 343, 347 (1964).

4. Kemula W., Buchowski H., Pawlowski W., Bull. Acad. Polon. Sci, Ser. sci. chim., 12, 491 (1964).

5. Kemula W., Buchowski H., Lew andowski R., Pawlowski W., Tepe r e k J., Bull. Acad. Polon. Sci., Ser. sci. chim., 14, 395 (1966).

6. Mottola H. A., Fre isner H., Talanta, 13, 55 (1966); 14, 864 (1967).

7. Коренман Я. И., Свнридов а Л. А., Тр. по химии и хим. технологии (Горь кий), вып. 2 (20), 122 (1968).

8. Vignes A., J. chim. phys. et phys.-chim. biol., 57, 966 (1960).

9. Fujita T., I w a s a J., H a n s ch C., J. Amer. Chem. Soc., 86, 5175 (1964).

10. H an sch C., Anderson S. M., J. Organ. Chem., 32, 2983 (1967).

11. Hansch C., Quinlan J. E., Law rence G. L., J. Organ. Chem., 33, 347 (1968).

12. Currie D. J., Longh C. E., Silver R. F., Holmes H. L., Canad. J. Chem., 44, 1035 (1966).

13. Hildebrand J. H., S cott R. L., The Solubility of Nonelectrolytes, 3rd Edn., Dover, New York, 1964.

14. Wakahay a shi T., Oki S., O mori T., Suzuki N., J. Inorg, and Nucl. Chem., 26, 2255 (1964).

15. O mori T., Wakahay a shi T., Oki S., Suzuki N., J. Inorg. and Nucl. Chem., 26,2265 (1964).

16. W a k a h a y a shi T., Bull. Chem. Soc. Japan, 40, 2836 (1967). 
17. Suzuki N., Akiba K., Kanno T., Wakahaya shi T., J. Inorg. and Nucl. Chem., 30, 2521 (1968).

18. Koj im a I., Y o sh id a M., T a n a k a M., J. Inorg. and Nucl. Chem., 32, 987 (1970).

19. Вайсбергер А., Проскауэр Э., Риддик Дж., Т у п Э., Органические растворители. М., 1958.

20. Коренман Я. И., Перешин В. В., Ж. прикл. химии, 43, 1410 (1970).

21. Коренман Я. И., С ви ридов а Л. А., Тр. по химии и хим. технологии (Горький), вып, 2 (20), 117 (1968).

22. Коренман И. М., Карякина Л. Н., Тр. по химии и хим. технологии (Горький), вып. 1 (19), 113 (1968).

23. Коренм ан И. М., Коче тков а Т. М., Тр. по химии и хим. технологии (Горький), вып. 2 (20), 110 (1968).

24. Коренм ан Я. И., Ж. прикл. химии, 43, 1100 (1970).

25. Коренм ан Я. И., Тр. по химии и хим. технологии (Горький), вып. 1 (19), 118 (1968)

26. H a n s e n C. M., Ind. Engng Chem., Product Research and Development, 8, 2 (1969).

27. Röck H., Rothe R., Z. phys. Chem. (Frankfurt), 12, 47 (1957).

Таллинский политехнический институт

Поступила в редакцию $4 / \mathrm{V} 1971$

\section{MOLDER, H. TAMVELIUS}

\section{FENOOLIDE JA AROMAATSETE AMIINIDE FAASIDEVAHELISE JAOTUMISE KIRJELDAMINE REGULAARLAHUSTE TEOORIA ABIL}

Artiklis esitatakse meetod iseassotsieeruvate ainete faasidevahelise jaotumise kirjeldamiseks regulaarlahuste teooria abil. Kõnesoleva meetodi kasutatavust kontrolliti ühe. aluseliste fenoolide ja aromaatsete amiinide jaotumise kaudu. Selleks määrati fenooli ning 3- ja 4-metüülfenoolide jaotuskoefitsiendid vee ja 15 orgaanilise lahusti vahel $20 \pm 1^{\circ} \mathrm{C}$ juures; kasutati ka kirjanduses avaldatud 2-metüülfenooli, 1-naftooli ja viie aromaatse amiini jaotusisoterme. Kōikide nende puhul leiti, et jaotuskoefitsient ja lahusti lahustuvusparameeter on omavahel heas korrelatsioonis. See meetod annab väga häid tulemusi siis, kui vee faasi lahustuvusparameetrina kasutada empiiriliselt leitud väärtusi (fenooli, 3 - ja 4-metüülfenoolide ja 1-naftooli jaotumisel on see 19,0,2-metüülfenooli jaotumisel 18,05, metüülfenüülamiinide jaotumisel 18,50 , naftüülamiinide jaotumisel 20,0 ), milledes on arvestatud interaktsiooni vee ja jaotuva aine vahel. Saadud andmete alusel arvutati jaotuvate ainete lahustuvusparameetrid.

\section{MOLDER, H. TAMVELIUS}

\section{APPLICATION OF THE REGULAR SOLUTION THEORY TO SOLVENT EXTRACTION OF PHENOLS AND AROMATIC AMINES}

The authors have made an attempt to extend the application of the regular solution theory to solvent extraction of self-associating substances. The validity of this treatment has been verified by presenting an example of monofunctional phenols and aromatic amines.

The partition coefficients of phenol and $m$ - and $p$-cresols between 15 inert organic solvents and an aqueous solution were determined at $20 \pm 1^{\circ} \mathrm{C}$, and the data were tabulated. To get more information for discussion, the published data on the partition of isotherms of $o$-cresol, $\alpha$-naphthol and five organic amines were recalculated. The limiting values of the partition coefficients at infinite dilution were then correlated quantitatively with the solubility parameters of the solvents. When an empirical value (19.0 for phenol, $m$ - and $p$-cresols and $\alpha$-naphthol, 18.05 for $o$-cresol, 18.50 for toluidines, 20.0 for naphthylamines) was employed as a solubility parameter of aqueous solution, the regular solution theory was confirmed to be distinctly valid. The solubility parameter for phenol was evaluated as $12.60 \pm 0.14, o$-cresol $-11.30 \pm 0.06, m$-cresol $-12.24 \pm 0.10, p$-cresol $12.23 \pm 0.09, \alpha$-naphthol $-11.76 \pm 0.10, o$-toluidine $-11.38 \pm 0.05, m$-toluidine $-11.42 \pm 0.05$, $p$-toluidine $-11.46 \pm 0.04, \alpha$-naphthylamine $-12.07 \pm 0.05, \beta$-naphthylamine $-12.08 \pm 0.05$. 